\title{
Sextant
}

Revue de recherche interdisciplinaire sur le genre et la sexualité

36 | 2019

No children, no cry

\section{Multiplier les enfants pour s'engager? Maternité de combat et dévotions. L'Archiconfrérie des Mères Chrétiennes (1860-1870)}

Azzurra Tafuro

\section{CpenEdition}

12 Journals

Édition électronique

URL : https://journals.openedition.org/sextant/351

DOI : 10.4000/sextant.351

ISSN : 2795-8736

Éditeur

Éditions de l'Université de Bruxelles

Édition imprimée

Date de publication : 1 décembre 2019

Pagination : 25-42

ISBN : 978-2-8004-1705-9

ISSN : 1370-267X

Référence électronique

Azzurra Tafuro, «Multiplier les enfants pour s'engager? Maternité de combat et dévotions.

L'Archiconfrérie des Mères Chrétiennes (1860-1870) », Sextant [En ligne], 36 | 2019, mis en ligne le 01 novembre 2021, consulté le 08 décembre 2021. URL : http://journals.openedition.org/sextant/351 ; DOI : https://doi.org/10.4000/sextant.351

\section{(c) (i) (ㅇ)}

La revue Sextant est mise à disposition selon les termes de la Licence Creative Commons Attribution Pas d'Utilisation Commerciale - Partage dans les Mêmes Conditions 4.0 International. 
PARTIE I

\section{(Dés)Enfanter}





\title{
Multiplier les enfants pour s'engager? Maternité de combat et dévotions L’Archiconfrérie des Mères Chrétiennes (1860-1870)
}

Azzurra TAFURO

\begin{abstract}
Résumé :
Il est difficile d'identifier des phénomènes de négation de la maternité au XIXe siècle - marqué par l'exaltation et le disciplinement de cette dernière. En même temps, plusieurs études ont souligné comment la réinterprétation de la maternité eut pour effet l'affaiblissement de certaines interdictions liées à l'existence des enfants, concernant par exemple l'accès des mères à la sphère publique et à la politique. Il suffit de penser aux réinterprétations opérées par les nationalismes du XIX siècle - telles que la Republican Motherhood aux États-Unis, ou les " mères patriotes" en Italie et en Allemagne, qui permettaient à beaucoup de femmes de participer intensément et activement aux processus de nation-building. Cet article porte sur le cas de l'Archiconfrérie des Mères Chrétiennes - un large groupe transnational catholique qui, multipliant symboliquement le nombre des enfants et faisant de la société entière l'objet des tâches de soin et d'éducation, fit de la maternité une forme originale de militance à la fois politique et religieuse. Dans cette stratégie, les dévotions eurent un rôle crucial, véhiculant des modèles légitimes et prestigieux. En se penchant sur les principales dévotions du groupe - Blanche de Castille, mère de Louis IX et Monique, mère d'Augustin d'Hippone -, il sera possible d'illustrer la maternité de combat des Mères Chrétiennes et, en même temps, de souligner les limites et les contradictions de leur projet.
\end{abstract}

\begin{abstract}
:
Rejection of motherhood was not common during the nineteenth century, when the maternal model was both exalted and disciplined. At the same time, many scholars have highlighted the fact that the reinterpretations of that model weakened some of the interdictions that women with children were subjected to-for example concerning access to the public sphere. The phenomenon is clearly shown by the reinterpretations of motherhood by nationalisms - the Republican Motherhood in the United States or the "patriots mothers" in Italy and in Germany, that allowed many women to take part in the nation-building process. This essay sheds light on the case of the Archiconfrérie des Mères Chrétiennes - a large transnational group of catholic women who made motherhood political by extending the maternal tasks (care, upbringing) from their children to the whole of society. Devotions were crucial in this strategy, providing role models that were both legitimate and prestigious. Focusing on the main devotions of the group, Blanche of Castille, mother of Louis IX, and Monica, mother of Augustin of Hippo, it will be possible to better understand the Mères Chrétiennes' militant motherhood, showing both the weaknesses and the strengths of their project.
\end{abstract}


Avant que les procédés techniques permettent de contrôler efficacement la fécondité, le choix pour les femmes de ne pas avoir d'enfant(s) se limitait à l'abstinence sexuelle ; seuls le voile et le célibat garantissaient contre les grossesses indésirables. De ce fait, longtemps, la maternité, quand elle n'était pas désirée, était conçue comme une fatalité, vécue au pire avec répugnance, angoisse ou anxiété, au mieux avec résignation. D'autant que, pour la société, les rôles, statuts et fonctions des femmes se définissaient proportionnellement à leur capacité reproductive ${ }^{1}$. Comment, dans une telle configuration, si l'on n'était ni religieuse ni célibataire, s'extraire de l'impératif à la fois biologique et social de la procréation ?

Il est difficile d'identifier des phénomènes de négation de la maternité au $\mathrm{XIX}^{\mathrm{e}}$ siècle - marqué par l'exaltation et le disciplinement de cette dernière. En même temps, plusieurs études ont souligné comment la réinterprétation de la maternité eut pour effet l'affaiblissement de certaines interdictions, concernant par exemple l'accès à la sphère publique et à la politique - ce qui aux yeux des critiques aurait pu détourner les mères de leurs enfants. Il suffit de penser aux réinterprétations opérées par les nationalismes du XIX ${ }^{\mathrm{e}}$ siècle - telles que la Republican Motherhood aux États-Unis, ou les « mères patriotes » en Italie et en Allemagne, qui - multipliant symboliquement le nombre des enfants et faisant de la société entière l'objet des tâches de soin et d'éducation - permettaient à beaucoup de femmes de participer intensément et activement aux processus de nation-building ${ }^{2}$. Ce travail se focalise sur l'Archiconfrérie des Mères Chrétiennes - un large groupe transnational catholique qui, suivant cette stratégie, fit de la maternité une forme originale de militance à la fois politique et religieuse. Le cas de l'Archiconfrérie semble confirmer l'intuition de Jean-Marie Mayeur qui, il y a presque vingt ans, invitait les spécialistes à examiner avec attention les élites féminines catholiques du XIX ${ }^{\mathrm{e}}$ siècle, en se disant convaincu que leurs déclarations officielles et leurs activités charitables cachaient un véritable engagement politique ${ }^{3}$.

${ }^{1}$ E. Berthiaud, « Grossesse désirée, grossesse imposée : le vécu de la grossesse aux $\mathrm{XVIII}^{\mathrm{e}}-\mathrm{XIX}{ }^{\mathrm{e}}$ siècles en France dans les écrits féminins privés », Histoire, économie \& société, 4, 2009, (28 année), p. 35-49.

2 Sur la Republican Motherhood voir L. Kerber, « The Republican Mother: Women and the Enlightenment. An American Perspective », American Quarterly, 2, 1976, p. 187-205 ; pour l'Italie voir : A. TAfuro, Madre e patriota. Adelaide Bono Cairoli, Firenze, Florence University Press, 2011 et " "Operaie della camicia rossa". Reti, pratiche e strategie della mobilitazione femminile nel 1866 », Memoria e Ricerca, 1, 2016, p. 127-146 ; pour l'Allemagne voir : U. Planert, « Vater Staat und Mutter Germania: Zur Politisierung des weiblichen Geschlechts im 19. und 20. Jahrhundert ", in U. Planert (hrsg.), Nation, Politik und Geschlecht: Frauenbewegungen und Nationalismus in der Moderne, Frankfurt, Campus Verlag, 2000, p. $15-65$.

3 J.-M. MAYeur, Les élites catholiques en France et en Allemagne de la fin du XIXe siècle à la fin de la Deuxième Guerre mondiale, in L. DuPEux (dir.), Élites en France et en Allemagne aux XIX et $X X^{\mathrm{e}}$ siècles. Structures et relations, vol. 2, Munich, Oldenburg Verlag, 1996, p. 188189. 
Cette invitation n'a été suivie d'effet qu'en partie. Si pour le $\mathrm{XX}^{\mathrm{e}}$ siècle Bruno Dumons et Magali Della Sudda ${ }^{4}$ ont mis en lumière les champs d'action et les enjeux des Ligues, pour le $\mathrm{XIX}^{\mathrm{e}}$ siècle en revanche, il reste beaucoup à faire, car les études se sont focalisées sur l'univers composite et riche des congrégations, sans s'attarder sur les profils des militantes et l'action de leurs groupes ${ }^{5}$. Ce traitement historiographique est bien illustré par le cas de l'Archiconfrérie des Mères Chrétiennes, resté quasiment inédit. À l'exception de Bonnie Smith qui s'est arrêtée brièvement sur le groupe de Lille, les chercheurs l'ont négligé, n'y voyant probablement qu'un simple groupe de mères dévotes ${ }^{6}$.

Pourtant l'association fondée à Lille en 1850 par Louise Josson ${ }^{7}$, une fervente catholique parisienne, eut bien un objectif politique : lutter contre le processus de sécularisation par la conversion de la société entière à la " véritable foi », à partir des pères, des fils et des époux qui s'en étaient éloignés ${ }^{8}$. Pour atteindre leur but, les Mères ne se limitèrent pas à la prière ou à la simple transmission de la foi, mais proches du mouvement intransigeant elles en épousèrent la cause et s'investirent sans relâche pour soutenir leur combat ${ }^{9}$. Ce n'est pas un hasard si leur militantisme s'intensifia dans les années 1850 et 1860, quand le processus d'unification de l'Italie menaça l'existence même des États Pontificaux et dégrada les relations entre les catholiques français et le régime. Pour défendre le pouvoir temporel du Pape, les associées encouragèrent leurs fils à s'enrôler et subventionnèrent le départ et l'armement des zouaves, constituant ainsi un réseau de soutien logistique et spirituel au volontariat blanc ${ }^{10}$.

${ }^{4}$ B. Dumons, Les Dames de la Ligue des Femmes françaises (1901-1914), Paris, Cerf, 2006 ; M. Della Sudda, « La politique malgré elles. Mobilisation féminines catholiques en France et en Italie (1900-1914) », Revue française de science politique, 60/1, 2010, p. 37-60.

${ }^{5}$ C. Langlois, Le catholicisme au féminin. Les congrégations françaises à supérieure générale au XIX $X^{\mathrm{e}}$ siècle, Paris, Cerf, 1984 ; S. CuRTIS, L'Enseignement au temps des congrégations. Le diocèse de Lyon (1801-1905), Lyon, Presses universitaires de Lyon, 2003.

${ }^{6}$ B. Smith, Ladies of the Leisure Class: The Bourgeoises of Northern France in the Nineteenth Century, Princeton, Princeton University Press, 1981.

7 Pour un profil biographique (mais hagiographique) voir : E. Humann de Chazelle, Une militante lä̈que. Louise Humann (1766-1836), Paris, Éd. Alsatia, 1957.

8 A. TAFuro, " "Anche le donne devono essere soldati". Cattoliche contro la "rivoluzione trionfante" ", Passato e Presente, 92, 2014, p. 31-54 ; A. TAFuro, " "Des femmes pleines de virilité". Les Mères Chrétiennes tra conversione e lotta alla modernità (1850-1870) », Mélanges de l'École française de Rome - Italie, et Méditerranée modernes et contemporaines, 128/2, 2016, p. 293-302.

${ }^{9}$ Une présentation synthétique du mouvement intransigeant français dans P. BoutRY, Ultramontanisme, in P. Levillain (dir.), Dictionnaire historique de la papauté, Paris, Fayard, 1994, p. 1651-1653.

10 J'ai développé ces considérations dans A. TAfuro, Une vocation guerrière. La mère des zouaves entre littérature et réalité, in C. VANDERPelen-Diagre, C. Sägesser (dir.), La Sainte Famille. Sexualité, filiation et parentalité dans l'Église catholique, Bruxelles, Éditions de l'Université de Bruxelles, 2017, p. 159-168. Sur le volontariat «blanc » voir au moins : C.E. Harrison, « Zouave Stories: Gender, Catholic Spirituality, and French Responses to the Roman Question », The Journal of Modern History, 2, 2007, p. 274-305 ; B. Dumons, H. Multon (dir.), Blancs et contre-révolutionnaires en Europe : espaces, réseaux, cultures et 
Peu d'années après la fondation, l'association connut un développement rapide. De nombreuses confréries virent le jour dans toute l'Europe (à Bruxelles en 1856 ; à Vienne en 1865 ; à Londres en 1867$)^{11}$; la France resta cependant le principal centre de diffusion. Aux côtés de celui de Paris, des groupes se démarquèrent par leurs effectifs comme celui d'Orléans, fort de 800 mères, d'Angers, avec 500 inscrites, de Marseille et de La Villette, avec respectivement 743 et 576 associées $^{12}$.

Les travailleuses vinrent grossir les rangs de l'association et, parfois, elles furent rassemblées dans des groupes distincts ${ }^{13}$. En voyant dans ces dernières les vecteurs de la possible rechristianisation des milieux ouvriers, le groupe leur proposa des modèles d'engagement respectueux de l'ordre social et du genre. Ce fut le cas d'Anna Maria Taïgi, la prophétesse-mère issue des classes populaires romaines, dont on soulignait la totale obéissance à l'autorité maritale dans la famille et à l'autorité ecclésiastique dans la société ${ }^{14}$.

Les véritables protagonistes de la vie de l'association furent les femmes des élites qui occupèrent les postes de direction. Par exemple, à ses débuts, la tête de la confrérie parisienne était composée de 18 associées, dont 11 étaient issues de l'aristocratie comme la princesse Octave de Broglie ou la vicomtesse Élie de Gontaut - viceprésidentes ${ }^{15}$. Il s'agissait de femmes avec un remarquable pouvoir politique et un réseau des relations large et prestigieux qu'elles pouvaient influencer très fortement.

De cette façon, elles devinrent les destinataires - mais également les artisanes d'un modèle de femme/mère catholique et militante fort novateur, qui devançait le tournant opéré par l'encyclique Rerum Novarum (1891). Dans la stratégie de réinterprétation de la maternité et de multiplication symbolique des enfants, les dévotions eurent un rôle crucial et devinrent un outil culturel et religieux par lequel les associées légitimèrent leur engagement ${ }^{16}$. Au cours des années 1860 , deux figures essentielles permirent aux Mères Chrétiennes de jouer un rôle actif dans deux scénarios

mémoires (fin XVIII ${ }^{e}$-début XIX $X^{e}$ siècles). France, Italie, Espagne, Portugal, Rome, Collection de l'École française de Rome, $n^{\circ}$ 454, 2011.

${ }^{11}$ Toutes les données relatives à la diffusion de l'association se rapportent à l'année 1868 et sont tirées de Anonyme, "Listes des confréries par diocèses ", Annales de l'Archiconfrérie de Mères Chrétiennes, 16, 1868, p. 764-770.

12 Ibid.

${ }^{13}$ Archives de Notre-Dame de Sion (dorénavant : ANDS), 2N9, Rapports et articles, L. Josson, Rapport fait à l'Archiconfrérie des Mères Chrétiennes [...], Paris, Olmer, p. 10.

14 J. Renard, «Anna Maria Taïgi », Annales de l'Archiconfrérie des Mères Chrétiennes, 12, 1868, p. 501-512. Sur la prophétesse : F. DE PALMA, « Il modello laicale di Anna Maria Taigi », in E. FATTORINI (éd.), Santi, culti, simboli nell'età della secolarizzazione (1815-1915), Rosenberg \& Sellier, Torino 1997, p. 529-546.

${ }^{15}$ Archiconfrérie des Mères Chrétiennes, Statuts et règlements [...], Paris, Remquet, 1856, p. 8.

${ }^{16}$ Sur la politisation des dévotions voir au moins D. Menozzi, Sacro Cuore. Un culto tra devozione interiore e restaurazione cristiana della società, Roma,Viella, 2001 et T. VAN Osselaer, « Home is where the Heart is. The Sacred Heart devotion in Catholic Families in Interwar Belgium », in T. Van Osselaer et P. Pasture (eds.), Christian Homes. Religion, Family and Domesticity in the $19^{\text {th }}$ and $20^{\text {th }}$ Centuries, Leuven, Leuven University Press, 2014, p. 159-178. 
cruciaux pour les catholiques : Blanche de Castille - qui fut leur modèle en France, où l'opposition des catholiques au régime se faisait toujours plus rude - et Monique, mère d'Augustin d'Hippone qui, par une récupération du mythe kabyle inspira leur action en Algérie, où les difficultés de la colonisation favorisèrent des relations plus cordiales avec les autorités de l'État qui laissaient entrevoir la possibilité d'établir une société « authentiquement chrétienne » aux yeux du mouvement intransigeant ${ }^{17}$.

\section{Blanche de Castille : une reine « virile " pour la conquête de la sphère publique}

Blanche n'a jamais été bienheureuse ni sainte, et pourtant les associées manifestèrent une grande dévotion envers elle, qui ne fut encouragée que plus tard par les autorités religieuses masculines. La première mention de la reine remonte à 1861, quand Josson recommanda vivement l'hagiographie rédigée par une consœur, Madame Orliac ${ }^{18}$ :

C'est l'histoire de la Reine Blanche de Castille, la mère de Saint Louis [...]. Le nom de l'auteur est également le pseudonyme d'une femme de grand mérite [...]. Vous me permettrez, Mesdames, de la remercier hautement d'avoir proposé à notre imitation un des plus beaux modèles que nous offrent les annales de l'histoire ${ }^{19}$.

L'hagiographie comptait parmi les rares ouvrages officiels de l'association et les termes d'admiration et de gratitude étaient réservés à son auteure. Dans son introduction, même Théodore Ratisbonne ${ }^{20}$, le charismatique directeur spirituel du groupe, qualifia l'auteure d'" habile et modeste ", apprécia l'ouvrage et en recommanda vivement la lecture ${ }^{21}$. Nous ne possédons que peu d'informations sur Orliac, mais les éloges dont elle fut l'objet ainsi que sa vaste production littéraire laissent à penser qu'elle provenait de la haute société et qu'elle faisait partie de l'élite de l'archiconfrérie ${ }^{22}$. Blanche de Castille, une figure fortement connotée d'un point de vue politique, dotée d'une forte personnalité et d'un esprit d'initiative, fut donc la dévotion que se donna un groupe de femmes influentes économiquement et socialement qui véhicula et légitima ainsi leur engagement politique et religieux, en l'étendant bien au-delà de la sphère privée et familiale. Dans cet ouvrage cependant, la reine n'en restait pas moins la mère de Louis IX, mais ses qualités politiques étaient

${ }^{17}$ O. SAAIDIA, Algérie coloniale. Musulmans et chrétiens : le contrôle de l'Etat (18301914), Paris, CNRS, 2015, p. 109-152.

${ }^{18}$ L'auteure publia sous le pseudonyme de J.M.S. Daurignac, voir G. D'HeYLLI, Dictionnaire des pseudonymes. Nouvelle édition entièrement refondue et augmentée, Paris, Dentu, 1887, p. 101.

${ }^{19}$ ANDS, 2N9, Rapports et articles, L. Josson, Rapport fait à l'Archiconfrérie [...], op. cit., p. 6.

${ }^{20}$ Pour un profil biographique voir : M.D. Gros, « Ratisbonne Théodore », in G. Pelliccia, G. Rocca (éds.), Dizionario degli Istituti di Perfezione, Milano, Edizioni Paoline, 1983, vol. 7, p. 1214-1215.

${ }^{21}$ T. Ratisbonne, "Introduction », in J.M.S. Daurignac, Blanche de Castille. Mère de Saint Louis et de Sainte Isabelle, Paris, Bray, 1861, p. 9-11.

${ }^{22}$ Voir par exemple J.M.S. Daurignac, Sainte Jeanne-Françoise de Chantal, modèle de la jeune fille et de la jeune femme dans le monde, et fondatrice de l'Ordre de la Visitation, Paris, Bray, 1858. 
reconnues par Louis VIII et Philippe II, lesquels ne manquèrent pas, selon Orliac, de la consulter à plusieurs reprises ${ }^{23}$. Ratisbonne reprit cette interprétation et, tentant une description ramassée du personnage, recourut à un paradoxe significatif : " Rien n'est plus admirable que le cœur de cette femme forte où l'on rencontre le merveilleux accord des qualités de l'homme d'État avec les plus tendres sollicitudes de la mère chrétienne $»^{24}$. Le directeur spirituel redessina le modèle de la femme forte - fondé sur l'interprétation littérale du chapitre XXXI des Proverbes - en le teintant d'une acception politique rare dans les traités de cette époque ${ }^{25}$. Parmi les principaux textes qui diffusèrent ce modèle, figurent l'essai de l'évêque de La Rochelle, Jean-Baptiste Landriot ainsi que le recueil de sermons que Mgr Félix Dupanloup adressa aux Mères entre 1862 et $1864^{26}$. Pourtant, les femmes fortes décrites restent bien différentes du modèle de Blanche de Castille : même si Landriot et Dupanloup accordèrent aux femmes catholiques un rôle clef dans le cadre familial, avec d'évidentes répercussions dans la sphère publique, ils ne leur reconnurent pas les capacités politiques soulignées par Ratisbonne. En définissant la reine comme un « homme d'État », il soutint que la force et la cohérence de son engagement politique furent telles qu'on pouvait les comparer à celles d'un homme. Pour le directeur, Blanche constitua une exception, capable de s'élever, par sa foi et sa lignée, au-dessus de la faiblesse qu'il considérait comme le trait caractéristique des femmes. Orliac partagea cette interprétation, en mettant en évidence la résolution avec laquelle la souveraine affronta les situations les plus difficiles : «Le ressort de cette politique exigeait de la prudence, de la temporisation, un coup d'œil pénétrant, de l'adresse, une inébranlable volonté. [...] Il semblait qu'une femme dût reculer devant une telle complication, mais [...] [elle] ne recula point $»^{27}$.

L'insistance sur le caractère exceptionnel de Blanche de Castille révèle que ce modèle n'était pas destiné à toutes les femmes, mais seulement à l'élite des Mères. Ces femmes, supérieures aux autres par leur classe et par leur foi, entendaient élargir et diversifier leur propre action politico-religieuse, en influençant la scène publique par une présence forte et efficace. Dans cet objectif, la figure maternelle et son devoir éducatif recouvraient un rôle clef; l'auteure s'attarda donc sur l'éducation de Louis IX, en représentant la souveraine en tant que conseillère politique ${ }^{28}$. Blanche devenait ainsi la femme qui avait formé le Roi saint de la France catholique ; l'éducation donnée était présentée comme éminemment politique, et visait à former un monarque catholique prêt à agir. En transposant ce modèle à son époque, Orliac se proposa de montrer comment de bonnes Mères chrétiennes pouvaient fournir à la France une

${ }^{23}$ Ibid., p. 78.

${ }^{24}$ T. RATISBONNE, «Introduction », op. cit., p. 9.

${ }^{25}$ Sur la femme forte voir D. Moulinet, « La femme forte. Modèle de la femme chrétienne », Chrétiens et sociétés, 15, 2008, p. 87-106.

${ }^{26}$ J.-B. Landriot, La femme forte. Conférences destinées aux femmes du monde, Paris, V. Palme, 1863 ; F. Dupanloup, Mgr Dupanloup aux femmes du monde. Entretiens recueillis par une associée des Mères Chrétiennes 1862-1864, Orléans, Herluison, 1880.

27 J.M.S. Daurignac, Blanche de Castille, op. cit., p. 111.

28 « Chier [sic] beau-fils [sic], êtes roi, devez être roi chrétien. [...] Chier Loys [sic], rois ne sont rien, sinon pour être le bras de Dieu dans le gouvernement du royaume » : ibid., p. 117. 
classe dirigeante composée d'hommes ayant une foi sûre, voués à la défense des intérêts de l'Église, et de fervents opposants à toute idée progressiste ou égalitaire susceptible de modifier la structure sociale. Les éducatrices d'une pareille classe dirigeante «méritaient » un espace politique plus vaste et une présence plus visible sur la scène publique. Leur requête s'appuyait à la fois sur leur origine sociale et sur leur rôle maternel et ne relevait en rien de la sphère du droit, car elle se présentait comme la défense et l'extension d'un privilège : seules les mères des élites pouvaient prétendre à de plus amples marges de manœuvre en termes d'action politique, mais aucunement les petites-bourgeoises ou les ouvrières qui ne seraient pas en mesure de procéder à la formation des classes dirigeantes.

Afin de mobiliser les aristocrates et les bourgeoises pour la défense des droits de l'Église, Ratisbonne encouragea le culte de Blanche en conservant la particularité de l'engagement politique, mais il en accentua la dimension de classe et le présenta comme étroitement lié à des causes contingentes. Dans son éloge de la vie de la reine, il s'attarda sur la lutte contre les barons, en l'insérant dans le projet divin d'une France unie et catholique. Dans son récit, la souveraine, décidée à faire « descendre sur ses peuples le règne de Dieu », était empêchée par les partisans du Duc de Bretagne qui projetaient de démembrer le pays ${ }^{29}$. La résistance acharnée la poussa à une intervention radicale :

Blanche, comme une héroïne, monte elle-même à l'assaut ; elle excite les soldats par sa bravoure intrépide ; elle partage leurs périls et leurs travaux ; elle renverse son fier ennemi, le relève et lui pardonne ; et enfin, calme dans son triomphe, elle rétablit le bon ordre dans ses états. [...] C'est ainsi qu'elle concourt à la réalisation du plan providentiel $^{30}$.

Dépeinte sur le champ de bataille, à l'assaut, occupée à encourager ses soldats et prête à partager avec eux les risques de l'affrontement, Blanche apporta sa contribution à l'ordre providentiel à travers sa participation directe au combat. Orliac souligna, elle aussi à plusieurs reprises, la présence de la régente sur le champ de bataille et justifia son absence à la croisade contre les Albigeois par l'approche de l'accouchement ${ }^{31}$.

Dans la figure de Banche, les qualités maternelles et les devoirs d'éducation cohabitent avec d'autres traits plus originaux, comme sa dimension guerrière qui caractérisait certaines représentations d'époque moderne. Par exemple, dans la biographie de Charles Combault Auteuil, Blanche est représentée avec les attributs de l'Archange Saint Michel, défenseur de la foi contre les légions de Satan. Dans le portrait, la reine comme ce dernier, apparaît au cœur de la bataille, tandis qu'elle brandit une lance et un bouclier, avec la tête de Méduse à ses pieds. L'image est surmontée d'une inscription significative : "Animos, curasque induta viriles » qui rappelle la définition oxymorique que lui donna Ratisbonne ${ }^{32}$.

${ }^{29}$ T. RATISBONNE, «Introduction », op. cit., p. 11.

${ }^{30}$ Ibid., p. 11-12.

31 J.M.S. Daurignac, Blanche de Castille, op. cit., p. 43-47.

32 C. De Combault Auteuil, Blanche, infante de Castille, mère de Saint Louis, reine et régente de France, Paris, de Sommaville, 1644. Cette devise apparaît également dans d'autres 


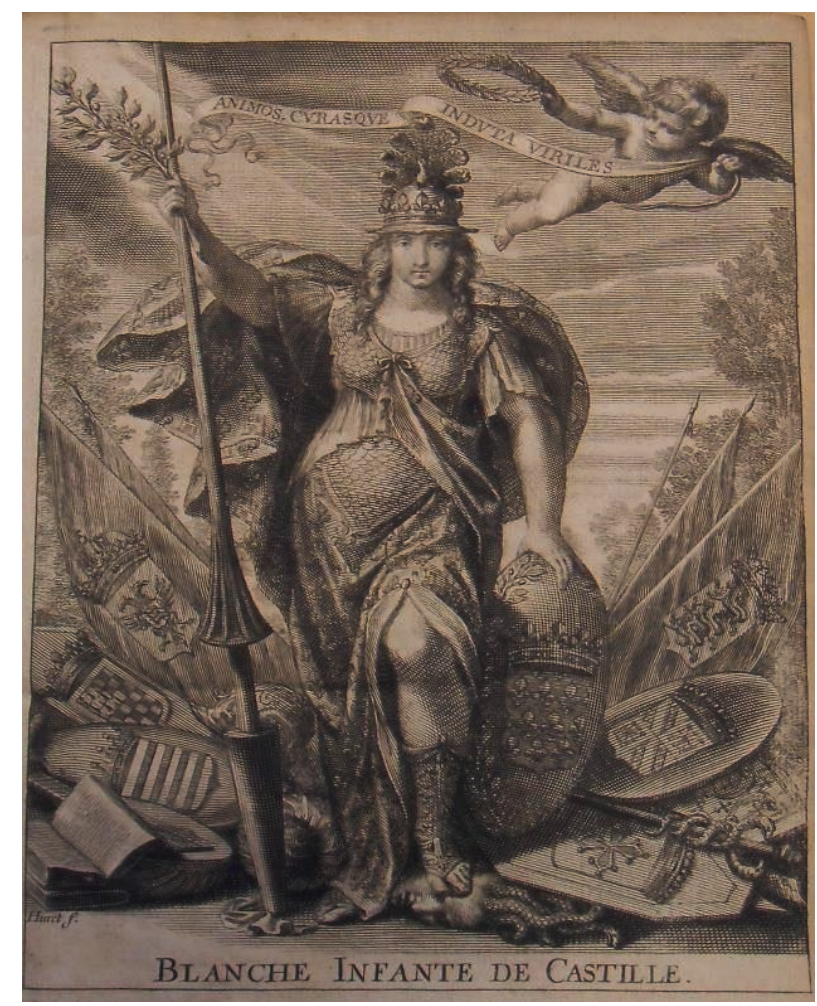

« Blanche Infante de Castille » ${ }^{33}$

Avec son interprétation de la souveraine, le directeur spirituel ne souhaita bien entendu pas inviter les associées à prendre réellement les armes, mais plutôt leur adresser une exhortation afin qu'elles soient plus actives sur la scène politique française - par une présence publique qu'il jugeait indispensable en ce moment critique que traversaient les catholiques. Ce n'est que quelques années plus tard qu'il revint sur ce point lors d'un sermon adressé à la direction de l'Archiconfrérie :

Quand au milieu des ténèbres les hommes cherchent leur chemin [...] une femme leur montre la route qu'elle n'a pas quittée [...]. Je vous rappelle les Clotilde, les Geneviève, les Blanche de Castille, [...] Mesdames, vous vivez dans le monde, au milieu d'un cercle plus ou moins étendu, vous pouvez éclairer par votre exemple et aussi par votre parole. Saint Paul défend aux femmes de prêcher, mais il ne leur défend de parler : si un mauvais [...] a dit des paroles que je n'accepte pas, je les proclame injustes [...]. On parle maintenant devant les femmes, et avec elles, de politique, de religion, de théologie, on s'entretient de Rome [...]. C'est alors, Mesdames, que vous pouvez et que vous devez parler : Saint Paul défend aux femmes la prédication, il ne leur défend pas la conversation. [...] Oh ! Mesdames, quand je me trouve en face des

hagiographies de la régente, voir par exemple : $\mathrm{M}^{\text {1le }}$ Vauvilliers, Histoire de Blanche de Castille, reine des Français, deux fois régente, Paris, Pauli, 1841, p. 179.

33 C. De Combault Auteuil, Blanche, infante de Castille, op.cit. 
femmes chrétiennes, comme vous l'êtes, je me sens pressé d'un ardent désir de voir en vous des femmes pleines de virilité pour attirer les cœurs et les conduire à Dieu ${ }^{34}$.

Conformément à un topos du catholicisme du XIX ${ }^{\mathrm{e}}$ siècle, Ratisbonne pensait que c'était aux femmes, restées intégralement catholiques même dans les bouleversements de la modernité, qu'il reviendrait de guider les hommes dans la lutte pour la défense des droits de l'Église ${ }^{35}$. Pour cette raison, il leur offrit en exemple une triade de saintes médiévales, qui se sont distinguées dans la défense du catholicisme contre le paganisme et l'hérésie : Clotilde, qui convertit Clovis et la France tout entière ; Geneviève, qui sauva Paris de l'invasion des barbares et Blanche de Castille ${ }^{36}$. En adaptant ces modèles pour la petite élite parisienne, Ratisbonne souligna qu'en tant que bonnes catholiques, elles devraient agir sur leur réseau de relations par l'exemple et la parole. La gravité de la situation politique le poussa à préciser l'interdiction de Paul qui, selon lui, avait certes interdit la prédication aux femmes, mais pas la conversation et le droit de parler en public. Par conséquent, les Mères pouvaient et devaient intervenir pour défendre le Pape et ses droits à chaque fois qu'ils seraient remis en cause, en dénonçant ouvertement l'iniquité de tels discours. En conclusion, il souhaita les voir affronter ce combat « pleines de virilité », à savoir avec la force, la vigueur, et la fermeté qu'il considérait propres à l'homme, en utilisant de nouveau un paradoxe pour définir le rôle plus large et plus actif qu'il avait conçu pour elles. Le recours à une expression appelant à la «virilité » chez les femmes rappelle la littérature chrétienne sur les martyres et suggère la conviction de Ratisbonne selon laquelle le catholicisme vivrait une nouvelle ère de persécution, semblable à celle des origines ${ }^{37}$. L'essor souhaité du rôle des femmes catholiques était donc circonscrit à ce moment de crise et revêtait la forme d'un devoir, celui du témoignage public de la foi contre le processus de sécularisation.

La « liquidité » d'un modèle intrinsèquement lié à la contingence est bien illustrée par la dernière représentation de Blanche de Castille établie par Ratisbonne. En 1866, ce dernier publia une nouvelle version de son manuel et inséra la souveraine parmi les mères exemplaires qui devraient inspirer les associées dans l'accomplissement de

${ }^{34}$ ANDS, 3j7, 2, Notes de Mme Josson de Bilhem. Instructions de Notre Père, février 1864.

${ }^{35}$ C. LANGLOIS, « Toujours plus pratiquantes. La permanence du dimorphisme sexuel dans le catholicisme français contemporain », Clio. Histoire, femmes, et sociétés, 2, 1995, p. 229260.

36 Sur Geneviève et Clotilde voir J. SCHMIDT, Le baptême de la France. Clovis, Clotilde, Geneviève, Paris, Seuil, 1996 et C. Thiellet, «La sainteté royale de Clotilde », in M. Rouche (dir.), Clovis, histoire et mémoire. Le baptême de Clovis, son écho à travers l'histoire, Paris, Presses de 1'Université Paris-Sorbonne, 1997, p. 147-156. Sur la dévotion à Sainte Geneviève voir : G. Cavagnini, A. Tafuro, Un passato che non torna. Il culto di Sainte Geneviève in Francia (1853-1918), in A. Mariuzzo, E. Mazzini, F. Mores, I. Pavan (dir.), « Un mestiere paziente ». Gli allievi pisani per Daniele Menozzi, ETS, Pisa, 2017, p. 67-92.

${ }^{37}$ La littérature chrétienne précisa souvent que les martyres avaient témoigné de leur foi en supportant de façon virile le supplice, voir : R. ZIADÉ, Les martyrs Maccabées de l'histoire juive au culte chrétien : les homélies de Grégoire de Nazianze et de Jean Chrysostome, Leiden, Brill, 2007, p. 246-252. 
leurs devoirs. La démarche était très différente des précédentes : traduit en plusieurs langues, le manuel était désormais destiné à un public plus large que la petite élite des Mères parisiennes. Ratisbonne ne mentionna pas l'ouvrage d'Orliac et donna une autre dimension à la figure de la souveraine qui « dirigeait avec une habilité ingénieuse et ferme le navire de l'État [...] sans qu'elle cessât un seul jour de remplir les devoirs de la maternité qui ne se prescrivent jamais $»^{38}$. La femme fut dépeinte comme une souveraine et une mère véritablement catholique, qui avait placé son peuple sous la direction de Dieu et lui avait offert ses fils. Ses qualités politiques ne furent pas niées, mais il ne restait en revanche aucune trace du caractère guerrier de la souveraine, dont on taisait l'engagement militaire et la présence sur le champ de bataille. C'est surtout dans sa conclusion que Ratisbonne ramena les Mères à leurs devoirs maternels :

Les mères chrétiennes comprendront, en étudiant ce beau modèle, que les exigences des positions les plus éminentes ne sauraient prévaloir sur les obligations intérieures de la famille ; et que, déléguées de Dieu auprès de leurs enfants, le plus saint de leurs devoirs, c'est de les élever chrétiennement. L'histoire de Saint Louis prouve que c'est par leurs enfants qu'elles seront à jamais honorées sur la terre et glorifiées au $\operatorname{ciel}^{39}$.

Dans la situation politique des catholiques, il ne s'était produit aucun bouleversement pouvant justifier le changement profond que subit le personnage, mais Ratisbonne, conscient de s'adresser à un public plus large, décida de dépouiller sa Blanche de tout caractère problématique ou controversé afin d'en faire un modèle pour toutes les Mères. Pour ce vaste groupe de femmes, la seule modalité d'intervention sur la société redevenait l'éducation catholique des enfants, devoir auquel personne ne pouvait se soustraire, pas même les élites, et qui assurerait à lui seul honneur et gloire sur terre et au ciel.

\section{Monique : une sainte kabyle pour participer à la francisation}

À la fin des années 1860, pour prendre part au processus d'assimilation et de conversion des Algériens, les Mères se servirent de la figure de Monique, une sainte berbère qui était également leur patronne et qui avait converti son fils Augustin. Leur engagement sur l'autre rive de la Méditerranée fut soutenu et encouragé par Mgr Félix de Las Cases, évêque de Constantine et d'Hippone ${ }^{40}$ qui, en 1869, proposait aux Mères de subventionner ses orphelinats. Faisant preuve d'une bonne connaissance du groupe, il évoqua Monique : « À Thagaste, où Sainte Monique a si longtemps pleuré, à Hippone, où les larmes de Monique ont porté si magnifiquement leurs fruits, deux chapelles vous sont dorénavant tout spécialement consacrées $»^{41}$.

${ }^{38}$ T. Ratisbonne, Nouveau manuel des mères chrétiennes, Paris, Poussielguer et Fils, 1866, p. 343.

${ }^{39}$ Ibid., p. 349.

${ }^{40}$ Sur de Las Cases voir : F. Combaluzier, L'Algérie catholique. 1830-1930 cent ans d'histoire religieuse dans l'Algérie française, in Paul LESOURD (éd.), L'année missionnaire 1931, Paris, Desclée de Brouwer, 1931, p. 375-395.

${ }^{41}$ ANDS, 2N8, Documents concernant l'CEuvre de S. Monique, Lettre de Mgr de Las Cases [...], s.l., s.d. [1869]. 
La consécration de deux chapelles dans des lieux hautement symboliques pour les associées leur était offerte en échange de leur soutien à un projet ambitieux :

J'ambitionne de nourrir, de loger, de vêtir, de moraliser, de christianiser, avec vos offrandes annuelles, mes innombrables et malheureux orphelins. [...] Sur le côteau [sic] d'Hippone va s'élever un orphelinat de jeunes filles [...] c'est sur vous que je compte pour l'accroître et pour l'aggrandir [sic]. Le R. P. Ratisbonne a accueilli mes propositions $[\ldots]$ comme le complément prévu de ses plans et de sa pensée ${ }^{42}$.

Conscient de l'importance de la conversion au sein de la mission des Mères, de Las Cases décrivit ses intentions - loger, vêtir, nourrir et surtout convertir les orphelines algériennes. En recourant à la figure de Monique, le prélat tenta de montrer aux associées que son dessein s'inscrivait en droite ligne avec leur mission : en effet, n'étaient-elles pas des dévotes de la sainte berbère ? Et les fillettes en question n'appartenaient-elles pas à la lignée d'Augustin et de sa mère ? L'évêque utilisa en quelque sorte Monique comme trait d'union entre l'Algérie et l'archiconfrérie et il offrit aux Mères la possibilité d'agir sur la terre de la sainte, même si c'était par personnes interposées en l'imitant par la conversion des orphelines. Ce lien fut renforcé par l'assentiment de Ratisbonne qui, au dire de Mgr de Las Cases, vit dans cette nouvelle entreprise la réalisation de la mission des Mères. De cette façon, la tâche de la conversion fut élargie et adaptée à l'esprit du temps : l'action en France devait apparaître insuffisante aux yeux du directeur spirituel tandis qu'à l'horizon, un nouveau peuple et un nouveau territoire se profilaient et constituaient, pour de nombreux intransigeants, un véritable laboratoire pour la construction d'une société « vraiment chrétienne $»^{43}$.

Dans le dessein de Mgr de Las Cases, les instances religieuses se mêlaient aux instances politiques, se fondant en une « mission civilisatrice». Les associées étaient invitées à y prendre part, pour faire de l'Algérie « une terre vraiment française, c'està-dire une terre chrétienne et civilisée ${ }^{44}$. Le projet soumis aux Mères reflétait cette double entreprise :

Je leur bâtis [...] des édifices où il me sera donné de veiller sur eux avec plus de sollicitude encore, en attendant que devenus chrétiens [...] ils contribuent plus tard à développer la colonisation française. [...] Je ferai comprendre aux Mères Chrétiennes l'importance d'une éducation homogène, forte et complète, pour cette jeunesse qui nous apparaît comme les prémisses des conquêtes de Jésus-Christ sur la vieille Terre des Fulgence, des Possidius, des Monique, des Augustin. [...] En considérant les premiers résultats obtenus, il nous semble que l'avenir est à nous. Quelle femme, quelle mère, ne voudrait dire : nous avons, en Afrique [...] des âmes qui, grâce à nos aumônes, connaissent Jésus-Christ et le servent? Quel citoyen, vraiment digne de ce

${ }^{42}$ Ibid..

${ }^{43}$ C. Prudhomme, Missions chrétiennes et colonisation $X V I^{e}-X X^{e}$ siècles, Paris, Cerf, 2004, p. $71-73$ et p. 96-108.

${ }^{44}$ ANDS, 2N8, Documents concernant l'CEuvre de S. Monique, Lettre de Mgr de Las Cases [...] adressée aux Membres de l'Archiconfrérie des Mères Chrétiennes [...], s.1., s.d. [1869]. 
nom, se refusera la consolation de participer à une œuvre d'humanité si profondément patriotique ${ }^{45}$ ?

Les rapports cordiaux entre l'État et l'Église en Algérie permirent au prélat de qualifier son projet de pieux et de patriotique, en affirmant qu'un citoyen français « vraiment digne de ce nom » ne pourrait lui refuser son soutien. Selon lui en effet, les Algériens convertis deviendraient des travailleurs pour donner leur contribution à la colonisation française. Leur éducation serait le premier pas vers la reconquête de la terre que de nombreux saints avaient donnée à l'Église : Possidius et Fulgence évêques de Calame et de Ruspe (Tunisie), Monique et Augustin. Dans ces figures de premiers chrétiens et de martyrs, il résuma l'histoire entière de l'Église d'Afrique, en surestimant délibérément l'expansion et l'enracinement. Son interprétation intégra des idées contenues dans le mythe kabyle, relancé dans les mêmes années par Mgr Charles Lavigerie - archevêque d'Alger - comme faisant partie intégrante d'un plus vaste projet de conversion et d'assimilation ${ }^{46}$. De Las Cases adapta ce schéma à ses besoins, en attribuant à Monique une centralité inédite et en faisant d'elle sa principale arme rhétorique pour rallier les Mères à son projet.

Entrevoyant la possibilité d'élargir leur engagement politique, les associées acceptèrent l'accord, inaugurant ainsi une collaboration qui se révéla fructueuse. Dès 1870 en effet, l'évêque écrivit à Josson pour lui dire que les orphelinats de Thagaste, de Constantine et d'Hippone « sont en pleine activité, et pourraient recevoir aisément 300 orphelins des deux sexes $»^{47}$. Cependant, à peine quelques mois plus tard, leur relation s'interrompit et de Las Cases, sans doute en raison de problèmes de santé, fut remplacé par Lavigerie. Ce remplacement s'accompagna de quelques changements, mais Monique et le mythe kabyle continuèrent à être utilisés pour mobiliser les Mères. Ce n'est pas pour rien que l'archevêque d'Alger mentionna la sainte dès sa première lettre :

Quoi de plus conforme [...] aux principes de la foi et de l'espérance catholiques, que de voir les Mères placer une plus vive confiance dans les prières qui s'élèvent de cette terre qui fut baignée des larmes de Monique [...] ? [...] Quoi de plus naturel que de voir un Évêque, le successeur même d'Augustin, s'associer à des sentiments aussi dignes des sympathies et du respect de toutes les âmes chrétiennes? N'est-ce pas un Évêque qui annonça à Monique que ses larmes sauveraient son fils ${ }^{48}$ ?

${ }^{45}$ ANDS, 2N8, Documents concernant l'Euvre de Sainte Monique, Félix Barthelemy de Las Cases à Théodore Ratisbonne, Aix, 10 février 1869.

${ }^{46}$ Sur Lavigerie voir : F. Renault, Le cardinal Lavigerie 1825-1892. L'Église, l'Afrique et la France, Paris, Fayard, 1992 ; sur le mythe kabyle voir C.-R. Ageron, Les Algériens musulmans et la France (1871-1919), Paris, PUF, 1968 ; P. LoRCIN, Kabyles, arabes, français : identités coloniales, Limoges, Pulim, 2005.

${ }^{47}$ ANDS, 2N8, Documents concernant l'CEuvre de Sainte Monique, Félix Barthelemy de Las Cases à Louise Josson, s.1., 30 mars 1870.

48 C. Lavigerie « Lettre de Mgr Lavigerie au très-révérend Père Théodore Ratisbonne », Euvre de Sainte Monique. Patronne des Mères Chrétiennes. Bulletin Trimestriel, 1, 1871, p. $1-2$. 
À l'instar de son prédécesseur, Lavigerie présenta l'Algérie comme la terre de Monique et proposa aux associées d'envoyer leurs offres au secrétaire de sa nouvelle structure, l'Euvre de Sainte Monique, auxquelles elles seraient automatiquement inscrites. L'objectif était de faire des Mères la base militante, dévote et puissante d'une œuvre destinée à soutenir économiquement et spirituellement son projet de conversion du continent entier.

Pour encourager les adhésions, Lavigerie accentua le rôle de Monique dans le mythe kabyle, proposé comme unique grille de lecture de la réalité coloniale comme en témoigne le bulletin de l'CEuvre qui s'adressa directement aux associées et accordait un rôle de grande importance à Monique et à Augustin :

La pieuse Monique, ressemblait à ces femmes des montagnes algériennes, que nous rencontrons nous-mêmes, car le type, les habitudes et jusqu'aux costumes de cette race se sont invariablement conservées dans cet orient africain, immeuble comme celui de l'Asie. Il m'est arrivé souvent, en voyant passer devant moi une de ces femmes accompagnées de son fils, de me dire en moi-même : c'était ainsi sans doute que Monique conduisait Augustin ${ }^{49}$.

Les Berbères et les Kabyles contemporains étaient représentés comme des émules des deux saints. À travers une superposition qui passait par la physionomie et les costumes, l'auteur put soutenir la continuité parfaite entre le $\mathrm{V}^{\mathrm{e}}$ siècle et le XIX ${ }^{\mathrm{e}}$ siècle. Ce trait forcé banalisait, dans une perspective raciste, l'histoire des Kabyles dans le but d'en promouvoir l'assimilation et la conversion. Dans cette optique, la pratique de l'Islam apparaissait comme le fruit des violentes persécutions arabes, qui avaient « contraint » les Kabyles à la conversion. Certains cependant avaient conservé le souvenir de leur ancienne foi :

Il y a quelques jours, j'interrogeai un des enfants kabyles, aujourd'hui chrétiens, qui se trouvent à l'orphelinat de Saint-Eugène. - T'a-t-on jamais dit, lui demandai-je, que vos ancêtres étaient catholiques. - Oui, me répondit-il, ma grand-mère me le disait souvent. - Et comment te disait-elle ? - Elle me disait : il y a bien longtemps, les Kabyles étaient comme les Français. Hélas ! Combien de Français ne sont plus dignes qu'on leur adresse un tel langage, et qu'on prenne leur nom pour synonyme de chrétiens ${ }^{50}$ !

Pensant que cela pouvait encourager les Mères à soutenir son œuvre, l'auteur rapporta le dialogue avec un jeune converti qui se rappelait les mots de sa grandmère sur les origines catholiques de son peuple. Le récit permettait de présenter le « rattachement » au catholicisme comme prémisse indispensable à l'assimilation et à la francisation des Kabyles ; les associées apparaissaient donc comme les patronnes idéales du projet, car elles s'occupaient déjà en France de ramener la société vers la foi.

Dans les pamphlets adressés aux Mères, les personnes les plus nécessiteuses les femmes, les personnes âgées et surtout les enfants - devinrent les personnages

${ }^{49}$ Anonyme, «Les descendants du peuple de saint Augustin et de sainte Monique », CEuvre de Sainte Monique. Patronne des Mères Chrétiennes. Bulletin Trimestriel, 2, 1872, p. 10-11.

${ }^{50}$ Ibid., p. 12. 
d'innombrables récits, conçus pour dissiper toute crainte de l'indigène méconnu et agressif. L'attention des auteurs se concentra principalement sur de jeunes Kabyles, considérés comme d'excellents candidats pour la francisation. Par exemple, l'abbé Lafettay, directeur de la confrérie de Bayeux, souligna la différence entre les adultes « paresseux, sensuels, fatalistes » et les enfants « reconnaissants, [...] naturellement religieux $\gg^{51}$; sur cette base, il encouragea les associées à soutenir l'CEuvre de Lavigerie, affirmant que « l'apostolat des enfants et surtout des orphelins est donc plein d'espérances. Cultivons ces natures ardentes ; arrachons-les à l'oisiveté, et nous en ferons, après quelques efforts, des agriculteurs intelligents, des travailleurs infatigables $\gg^{52}$. Il ne s'agissait pas seulement de solliciter l'instinct maternel des associées, mais plutôt de les pousser à l'action en leur montrant le potentiel de la jeunesse. Si en effet, des soupçons d'opportunisme ou de double dévotion pesaient sur les adultes, le jeune âge et la faible pratique de l'Islam faisaient des enfants de parfaits néophytes qu'il était aisé de former aux principes catholiques. Ainsi, lors d'un séjour en Europe pour participer au Concile, un homme aussi avisé que Lavigerie se fit accompagner par trois orphelins issus de ses instituts ${ }^{53}$. Dans une attitude qui rappelle les expositions coloniales de l'époque, l'archevêque amena devant Pie IX ses premiers Algériens convertis au catholicisme comme preuve de son succès. L'un d'entre eux, Paul, poursuivit le voyage avec Lavigerie et rendit hommage aux Mères de Bayeux, ses bienfaitrices. En se remémorant cet épisode, Lafettay salua dans le jeune Kabyle «l'apôtre de ses compatriotes » et annonça que cinquante orphelins souhaitaient déjà suivre ses pas, tout en se demandant : «Qui sait si de cette pépinière il ne sortira pas pour l'Algérie un germe de salut ? ${ }^{54}$ Cette rencontre avec Lavigerie et son protégé arriva à un moment où les Mères étaient en train de perdre leur rôle de principal soutien de l'EEuvre, comme en témoigne le bulletin qui progressivement ne relate plus d'information à leur sujet. La dernière et furtive mention de l'archiconfrérie apparaît en 1874 quand les présidentes des confréries qui n'étaient pas encore inscrites à l'Euvre furent invitées à régulariser leur situation ${ }^{55}$. En l'absence d'informations ultérieures, on peut supposer que la quasi-totalité des associées françaises avait aussi adhéré à l'Euvre. Cette dernière, qui initialement s'était développée en se superposant quasiment au groupe, avait ensuite connu une forte expansion et reçu de multiples approbations. Les Mères étaient ainsi devenues de simples inscrites et le bulletin avait cessé de les considérer comme leurs principales bienfaitrices.

\section{Conclusion}

En conclusion, il me semble important de réfléchir sur une stratégie qui ne refusa jamais la maternité mais qui la réinterpréta et l'élargit à la société entière ayant pour effet d'affaiblir les interdictions liées à l'existence des enfants biologiques. Pourtant,

${ }^{51}$ C.J. Lafettay, Euvre de Mgr Lavigerie Archevêque d'Alger, Discours adressé aux Mères Chrétiennes de Bayeux [...], Bayeux, H. Grobon et O. Payan, 1870, p. 11.

${ }_{52}$ Ibid., p. 14.

${ }^{53}$ F. Renault, Le cardinal Lavigerie 1825-1892, op. cit., p. 161.

${ }^{54}$ C.J. Lafettay, Euvre de Mgr Lavigerie Archevêque d'Alger, Discours, op. cit., p. 15.

${ }_{55}$ Anonyme, «Avis », Euvre de Saint-Augustin et de Sainte-Monique patronne des Mères Chrétiennes sous la protection de Notre-Dame d'Afrique. Bulletin trimestriel, 9, 1874, p. 2. 
on ne peut pas nier que cette stratégie, quoiqu'originale, eut des limites importantes - soulignées par les dévotions analysées. Fortes de cette maternité réinterprétée et de ces interdictions devenues plus faibles, les associées pouvaient se battre en faveur d'un projet de société précis, sans courir le risque du blâme public, car leur action s'inscrivait dans une sphère d'action autorisée. Par ailleurs, il ne faut pas sous-estimer le fait que les inflexions données à la dévotion pour Blanche de Castille et pour Sainte Monique illustrent combien les Mères partageaient l'idée profondément enracinée dans le mouvement intransigeant, d'une société rigoureusement ordonnée selon la classe, le genre et l'ethnie. À travers leur réinterprétation et leur action les associées ne contestèrent pas cet ordre, ses limites et ses interdictions, mais contribuèrent au contraire à les renforcer : elles ne revendiquèrent jamais le droit à l'action politique pour toutes les femmes mais plutôt défendirent le privilège des femmes des élites de contourner les interdictions et les restrictions liées à la maternité et de faire de la politique. De cette façon, la majorité des femmes restait exclue de la sphère publique et condamnée à la minorité politique et juridique.

Le prolongement au $\mathrm{XX}^{\mathrm{e}}$ siècle - non des moindres - des cultes analysés met en lumière l'héritage politique de l'archiconfrérie en le reliant aux Ligues. Il ne faut donc pas s'étonner qu'en 1905, au cours de l'un des moments les plus critiques pour le catholicisme français, Blanche de Castille et sa dévotion combattante redevinrent un modèle pour les femmes des élites catholiques. En s'adressant aux associées de la Ligue des Femmes Françaises, le cardinal de Lyon, Pierre-Hector Coullié, les incita à se mobiliser contre la Séparation de l'Église et de l'État en évoquant « [des] Geneviève, [des] Clotilde, [des] Blanche de Castille, [...] et tant d'autres héroïnes dont l'histoire nous redit l'heureuse influence pour le salut de la Patrie ${ }^{56}$, à savoir les mêmes modèles qui, cinquante ans plus tôt, avaient inspiré et légitimé la militance des Mères.

\section{Bibliographie}

Ageron C.-R., Les Algériens musulmans et la France (1871-1919), Paris, PUF, 1968. Anonyme, "Listes des confréries par diocèses », Annales de l'Archiconfrérie de Mères Chrétiennes, 16, 1868, p. 764-70.

Anonyme, "Les descendants du peuple de saint Augustin et de sainte Monique ", Euvre de Sainte Monique. Patronne des Mères Chrétiennes. Bulletin Trimestriel, 2, 1872, p. 10-11.

Anonyme, "Avis », Euvre de Saint-Augustin et de Sainte-Monique patronne des Mères Chrétiennes sous la protection de Notre-Dame d'Afrique. Bulletin trimestriel, 9, 1874, p. 2.

ArChiCONFrérIe des Mères ChrétIENnes, Statuts et règlements de l'Archiconfrérie des Mères Chrétiennes, Paris, Remquet, 1856.

Berthiaud E., "Grossesse désirée, grossesse imposée : le vécu de la grossesse aux XVIII ${ }^{\mathrm{e}}-\mathrm{XIX}^{\mathrm{e}}$ siècles en France dans les écrits féminins privés », Histoire, économie \& société, 4, 2009, p. 35-49.

${ }^{56}$ B. Dumons, Les Dames de la Ligue des Femmes françaises, op. cit., p. 330. 
Boutry P., Ultramontanisme, in Levillain P. (dir.), Dictionnaire historique de la papauté, Paris, Fayard, 1994, p. 1651-1653.

Cavagnini G., Tafuro A., Un passato che non torna. Il culto di Sainte Geneviève in Francia (1853-1918), in Mariuzzo A., Mazzini E., Mores F., Pavan I. (dir.), «Un mestiere paziente». Gli allievi pisani per Daniele Menozzi, ETS, Pisa, 2017, p. 67-92.

COMbaluzier F., L'Algérie catholique. 1830-1930 cent ans d'histoire religieuse dans l'Algérie française, in LESOURD P. (dir.), L'année missionnaire 1931, Paris, Desclée de Brouwer, 1931, p. 375-395.

Combault Auteuil (DE) C., Blanche, infante de Castille, mère de Saint Louis, reine et régente de France, Paris, de Sommaville, 1644.

Curtis S., L'Enseignement au temps des congrégations. Le diocèse de Lyon (18011905), Lyon, PUL, 2003.

D’Heylli G., Dictionnaire des pseudonymes. Nouvelle édition entièrement refondue et augmentée, Paris, Dentu, 1887.

Daurignac J.M.S., Sainte Jeanne-Françoise de Chantal, modèle de la jeune fille et de la jeune femme dans le monde, et fondatrice de l'Ordre de la Visitation, Paris, Bray, 1858.

Daurignac J.M.S., Blanche de Castille. Mère de Saint Louis et de Sainte Isabelle, Paris, Bray, 1861.

De Palma F., Il modello laicale di Anna Maria Taigi, in Fattorini E. (dir.), Santi, culti, simboli nell'età della secolarizzazione (1815-1915), Torino, Rosenberg \& Sellier, 1997, p. 529-546.

Della Sudda M., « La politique malgré elles. Mobilisation féminines catholiques en France et en Italie (1900-1914) », Revue française de science politique, 60/1, 2010, p. 37-60.

Dumons B., Les Dames de la Ligue des Femmes françaises (1901-1914), Paris, Cerf, 2006.

Dumons B., Multon H. (dir.), Blancs et contre-révolutionnaires en Europe : espaces, réseaux, cultures et mémoires (fin XVIII ${ }^{e}$-début XIX ${ }^{e}$ siècles). France, Italie, Espagne, Portugal, Rome, Collection de l'École Française de Rome, n 454 , 2011.

Dupanloup F., Mgr Dupanloup aux femmes du monde. Entretiens recueillis par une associée des Mères Chrétiennes. 1862-1864, Orléans, Herluison, 1880.

Gros M.D., Ratisbonne Théodore, in Pelliccia G., Rocca G. (dir.), Dizionario degli Istituti di Perfezione, Milano, Edizioni Paoline, 1983, vol. 7, p. 1214-1215.

Harrison C.E., « Zouave Stories: Gender, Catholic Spirituality, and French Responses to the Roman Question », The Journal of Modern History, 2, 2007, p. 274-305.

Josson L., Rapport fait à l'Archiconfrérie des Mères Chrétiennes dans la chapelle de Notre-Dame de Sion par Mme Louise Josson Présidente, Assemblée générale du 8 décembre 1859, Paris, Olmer, 1859.

Kerber L., " The Republican Mother: Women and the Enlightenment. An American Perspective », American Quarterly, 2, 1976, p. 187-205.

Lafettay C.J., Euvre de Mgr Lavigerie Archevêque D’Alger, Discours adressé aux Mères Chrétiennes de Bayeux le jour de la fête de Saint Joseph (mars 1870) 
par l'Abbé J. Laffettay, chanoine de Bayeux, Bayeux, H. Grobon et O. Payan, 1870.

Landriot J.-B., La femme forte. Conférences destinées aux femmes du monde, Paris, V. Palme, 1863.

LANGLOIS C., Le catholicisme au féminin. Les congrégations françaises à supérieure générale au XIX' siècle, Paris, Cerf, 1984.

Langlois C., « Toujours plus pratiquantes. La permanence du dimorphisme sexuel dans le catholicisme français contemporain », Clio. Histoire, femmes, et sociétés, 2, 1995, p. 229-260.

Lavigerie C., " Lettre de Mgr Lavigerie au très-révérend Père Théodore Ratisbonne », Euvre de Sainte Monique. Patronne des Mères Chrétiennes. Bulletin Trimestriel, $1,1871$.

Lorcin P., Kabyles, arabes, français : identités coloniales, Limoges, Pulim, 2005.

Madeleine Louise de Sion, Edith Humann de Chazelle, Une militante laïque. Louise Humann (1766-1836), Paris, Éd. Alsatia, 1957.

Mayeur J.-M., Les élites catholiques en France et en Allemagne de la fin du $X I X^{e}$ siècle à la fin de la Deuxième Guerre mondiale, in Dupeux L. (dir.), Élites en France et en Allemagne aux XIX et XX $X^{e}$ siècles. Structures et relations, vol. 2, Munich, Oldenburg Verlag, 1996, p. 185-194.

D. Menozzi, Sacro Cuore. Un culto tra devozione interiore e restaurazione cristiana della società, Roma,Viella, 2001.

Planert U., Vater Staat und Mutter Germania: Zur Politisierung des weiblichen Geschlechts im 19. und 20. Jahrhundert, in Planert U. (hrsg.) Nation, Politik und Geschlecht: Frauenbewegungen und Nationalismus in der Moderne, Frankfurt, Verlag, 2000, p. 15-65.

Prudhomme C., Missions chrétiennes et colonisation $X V I^{e}-X X^{e}$ siècle, Paris, Cerf, 2004.

Ratisbonne T., Nouveau manuel des mères chrétiennes, Paris, Poussielguer et Fils, 1866.

Renard J., « Anna Maria Taïgi », Annales de l'Archiconfrérie des Mères Chrétiennes, 12,1868 , p. 501-512.

Renault F., Le cardinal Lavigerie 1825-1892. L'Église, l'Afrique et la France, Paris, Fayard, 1992.

SAaidia O., Algérie coloniale. Musulmans et chrétiens : le contrôle de l'État (18301914), Paris, CNRS, 2015.

Schmidt J., Le baptême de la France. Clovis, Clotilde, Geneviève, Paris, Seuil, 1996.

Sмiтн B., Ladies of the Leisure Class: The Bourgeoises of Northern France in the Nineteenth Century, Princeton, Princeton University Press, 1981.

Tafuro A., Madre e patriota. Adelaide Bono Cairoli, Firenze, Florence University Press, 2011.

TAFuro A., «"Anche le donne devono essere soldati”. Cattoliche contro la "rivoluzione trionfante" ", Passato e Presente, 92, 2014, p. 31-54.

TAfuro A., " "Operaie della camicia rossa". Reti, pratiche e strategie della mobilitazione femminile nel $1866 »$, Memoria e Ricerca, 1, 2016, p. 127-146. 
TAfuro A., « "Des femmes pleines de virilité”. Les Mères Chrétiennes tra conversione e lotta alla modernità (1850-1870) », Mélanges de l'École française de RomeItalie, et Méditerranée modernes et contemporaines, 128/2, 2016, p. 293-302.

TAFURO A., "Une vocation guerrière. La mère des zouaves entre littérature et réalité », in Vanderpelen-Diagre C., Sägesser C. (dir.), La Sainte Famille. Sexualité, filiation et parentalité dans l'Église catholique, Bruxelles, Éditions de l’Université de Bruxelles, 2017, p. 159-168.

Thiellet C., « La sainteté royale de Clotilde », in Rouche M. (dir.), Clovis, histoire et mémoire. Le baptême de Clovis, son écho à travers l'histoire, Paris, Presses de l’Université de Paris-Sorbonne, 1997, p. 147-156.

Van Osselaer T., «Home is where the Heart is. The Sacred Heart devotion in Catholic Families in Interwar Belgium », in Van Osselaer T., Pasture P. (eds.), Christian Homes. Religion, Family and Domesticity in the $19^{\text {th }}$ and $20^{\text {th }}$ Centuries, Leuven, Leuven University Press, 2014, p. 159-178.

Vauvilliers $\mathrm{M}^{\text {lle }}$, Histoire de Blanche de Castille, reine des Français, deux fois régente, Paris, Pauli, 1841.

ZiAdÉ R., Les martyrs Maccabées de l'histoire juive au culte chrétien : les homélies de Grégoire de Nazianze et de Jean Chrysostome, Leiden, Brill, 2007. 